\title{
Bayesian Network Classifiers for Categorizing Cortical GABAergic Interneurons
}

\author{
Bojan Mihaljević • Ruth Benavides-Piccione • \\ Concha Bielza $\cdot$ Javier DeFelipe $\cdot$ Pedro Larrañaga
}

\begin{abstract}
An accepted classification of GABAergic interneurons of the cerebral cortex is a major goal in neuroscience. A recently proposed taxonomy based on patterns of axonal arborization promises to be a pragmatic method for achieving this goal. It involves characterizing interneurons according to five axonal arborization features, called F1-F5, and classifying them into a set of predefined types, most of which are established in the literature. Unfortunately, there is little consensus among expert neuroscientists regarding the morphological definitions of some of the proposed types. While supervised classifiers were able to categorize the interneurons in accordance with experts' assignments, their accuracy was limited because they were trained with disputed labels. Thus, here we automatically classify interneuron subsets with different label reliability thresholds (i.e., such that every cell's label is
\end{abstract}

backed by at least a certain (threshold) number of experts). We quantify the cells with parameters of axonal and dendritic morphologies and, in order to predict the type, also with axonal features F1-F4 provided by the experts. Using Bayesian network classifiers, we accurately characterize and classify the interneurons and identify useful predictor variables. In particular, we discriminate among reliable examples of common basket, horse-tail, large basket, and Martinotti cells with up to $89.52 \%$ accuracy, and single out the number of branches at $180 \mu \mathrm{m}$ from the soma, the convex hull 2D area, and axonal features F1-F4 as especially useful predictors for distinguishing among these types. These results open up new possibilities for an objective and pragmatic classification of interneurons.

Keywords Neuronal classification · Morphological features · Label reliability · Multiple annotators · Weighted naive Bayes

\section{Introduction}

GABAergic interneurons of the cerebral cortex are highly diverse with regard to their morphological, physiological and molecular features (Ascoli et al. 2008). The scientific community considers that this variability indicates that different classes of interneurons exist and that the differences between classes are functionally relevant. Although a number of classification schemes have been proposed since the times of Santiago Ramón y Cajal (e.g., Fairén et al. 1992; Kawaguchi 1993; Cauli et al. 1997; Somogyi et al. 1998; Gupta et al. 2000; Maccaferri and Lacaille 2003), there is no catalogue of interneuron types accepted by the scientific community (DeFelipe et al. 2013), making it hard to organize data and share knowledge. The Petilla terminology 
(Ascoli et al. 2008) provided a stepping stone towards a future classification by standardizing the nomenclature of features that distinguish types of GABAergic interneurons. However, the morphological, physiological, and molecular classification schemes derived from the terminology are incomplete on their own while their comprehensive combination is difficult to realize in practice (DeFelipe et al. 2013).

Thus, as a pragmatic alternative, DeFelipe et al. (2013) proposed a taxonomy based mainly on patterns of axonal arborization. It involves characterizing interneurons according to five axonal arborization features, such as the distribution of the arbor with respect to the soma's layer, as well as classifying them into a set of predefined types, most of which are established in the literature. The authors sought community-wide consensus on the taxonomy and they, therefore, asked 42 expert neuroscientists to categorize a representative set of interneurons. Unfortunately, there was little inter-expert agreement on the morphological definitions of some of the proposed types; however, there was, in general, consensus on the definitions of the axonal features. As a positive outcome, supervised classifiers were able to categorize the interneurons in accordance with the experts' assignments. Nonetheless, they were inaccurate at predicting the interneuron type and one of the axonal features; the former result being reasonable since distinguishing interneuron types was hard even for the experts. The classifiers were trained by labelling each cell with the category that was most commonly selected by the experts (majority vote). While this is a common approach in supervised classification with multiple annotators (Raykar et al. 2010) it allowed labels that were backed by few experts (i.e., 'weak majorities', those due to very ambiguous categorizations) to be used as true labels.

Thus, in this paper, we approach supervised classification of interneurons by using reliably labeled cells alone. The rationale is that unreliable labels would confuse a classifier and that it would be better to have them assigned by a model trained with reliable labels. One possibility would be to remove unreliable labels (i.e., unlabel instances) and cluster the data in a semi-supervised fashion, similarly to Mihaljević et al. (2014), allowing the discovery of previously unknown types. Here we classify reliably labeled cells alone, in a supervised fashion, seeking to discover whether at least a subset of interneurons can be accurately categorized. Rather than proposing a criterion for discerning reliable labels from unreliable ones, we form many data subsets with different label reliability thresholds (i.e., such that each instance's label is backed by at least a certain (threshold) number of experts) and analyze the supervised classification of each data subset separately.
We separately categorize the interneurons according to interneuron type and four axonal features, ${ }^{1}$ termed F1, F2, F3, and F4 (see 'Materials and Methods' for details). We measure 214 parameters of axonal and dendritic arborizations and use all or some of them as predictive variables, depending on the axonal feature/type to be predicted. Additionally, we use axonal features F1-F4 as predictors of the interneuron type, both on their own and together with the morphological parameters. We estimate their crisp (i.e., atomic) values with majority vote, thus discarding, when using axonal features $\mathrm{F} 1-\mathrm{F} 4$ as predictors, interneurons unreliably categorized according to at least one of those features. Figure 1 shows an overview of the described supervised classification tasks.

We tackle each classification task with four different Bayesian network classifiers (Bielza and Larrañaga 2014). These are competitive performance classifiers (Morales et al. 2013; Friedman et al. 1997) that allow for analyzing probabilistic relationships among the variables of a domain and, among other desirable properties, accommodate for feature selection, can fit complex labelling scenarios-such as missing or partial labels; multiple class variablesand can be efficiently learned from data (see Bielza and Larrañaga 2014).

The rest of this paper is organized as follows. 'Materials and Methods' describes the data and the practical approach to interneuron classification and then elaborates on the methodology - the formation of data sets according to label reliability; the Bayesian network classifiers used; data preprocessing; and the empirical setup. 'Results' presents the results and 'Conclusions' rounds off with conclusions.

\section{Materials and Methods}

\section{Data}

All neurons and experts' terminological choices were gathered by DeFelipe et al. (2013). We used 241 cells from different areas and layers of the cerebral cortex of the mouse, rat, and monkey, whose digital reconstructions were obtained from NeuroMorpho.Org (Ascoli et al. 2007). 40 digital reconstructions exhibited an interrupted axonal process; if deemed feasible ( 36 cells), we drew the small missing fragments using the Neurolucida workstation (Glaser and Glaser 1990), thus completing the axon. Due to nontrivially reconstructible axons four cells were omitted from our study, yielding the final sample of 237 cells.

\footnotetext{
${ }^{1}$ The prediction of one of the features, F6, is almost trivial and was thus not considered here.
} 


\begin{tabular}{c|c|c}
$\mathcal{D}_{25}^{5}$ & $X_{1}, \ldots, X_{214}$ & $\mathrm{~F} 5$ \\
\hline 2 & $0.2, \ldots, 4.1$ & MA \\
3 & $1.2, \ldots, 4.2$ & HT \\
$\ldots$ & $\ldots$ & $\ldots$ \\
237 & $1.0, \ldots, 2.2$ & CB
\end{tabular}
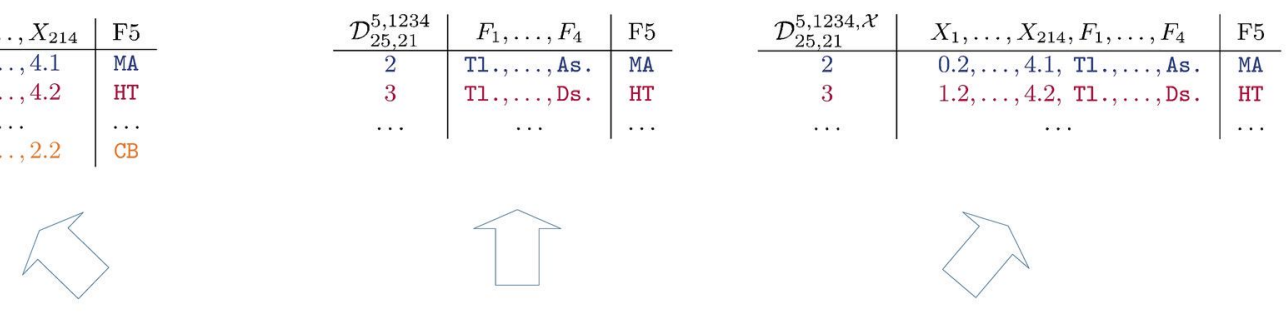

\begin{tabular}{c|c|c|c|c|c|c}
$\mathcal{D}$ & $X_{1}, \ldots, X_{214}$ & $\mathrm{~F} 1$ & $\mathrm{~F} 2$ & $\mathrm{~F} 3$ & $\mathrm{~F} 4$ & $\mathrm{~F} 5$ \\
\hline 1 & $0.5, \ldots, 2.1$ & Il. (30) & Ic. $(30)$ & Ce. (30) & Bo. (10) & CT (21) \\
2 & $0.2, \ldots, 4.1$ & Tl. (40) & Tc. (29) & De. (40) & As. (39) & MA (40) \\
3 & $1.2, \ldots, 4.2$ & Tl. (40) & Ic. (39) & De. (40) & Ds. (40) & HT (40) \\
$\ldots$ & $\ldots$ & $\ldots$ & $\ldots$ & $\ldots$ & $\ldots$ & $\ldots$ \\
237 & $1.0, \ldots, 2.2$ & Il. (21) & Ic. $(24)$ & Ce. (35) & As. (7) & CB $(26)$
\end{tabular}
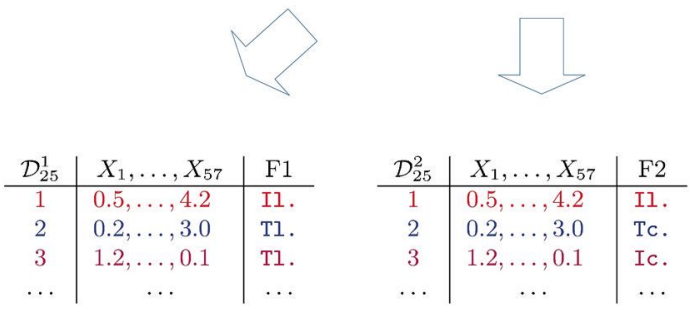

Fig. 1 A schematic overview of our automatic categorization of interneurons according to type and axonal features F1-F4. The full data set, $\mathscr{D}$ (center), is used to form data subsets (shown around $\mathscr{D}$ ) with different class variables (indicated by the superscript; e.g., F1 is the class variable in $\mathscr{D}_{25}^{1}$ ), predictor variables (second and third superscript; e.g., $\mathscr{D}_{25,21}^{5,1234}$ has features F1-F4 as predictors of the type), label reliability threshold (first subscript; e.g., $\mathscr{D}_{25}^{1}$ has label reliability 25 ), and predictor reliability threshold (second subscript; e.g., $\mathscr{D}_{25,21}^{5,1234}$ has reliability 21 for predictors $\mathrm{F} 1-\mathrm{F} 4$ ). In $\mathscr{D}$, all instances are quantified with 214 morphological parameters and labeled with the majority vote for the interneuron type and each axonal feature, with the label reliability (number of agreeing experts) shown in parentheses. The predictive

\section{Axonal Feature-Based Nomenclature}

The interneuron nomenclature proposal by DeFelipe et al. (2013) consists of six categorical features of axonal arborization. These features have the following categories:

- Feature 1 (F1): intralaminar and translaminar

- Feature 2 (F2): intracolumnar and transcolumnar

- Feature 3 (F3): centered and displaced

- Feature 4 (F4): ascending, descending, and both

- Feature 5 (F5): arcade (AR), Cajal-Retzius (CR), chandelier (CH), common basket (CB), common type (CT), horse-tail (HT), large basket (LB), Martinotti (MA), neurogliaform (NG), and other (OT)

- Feature 6 (F6): characterized and uncharacterized
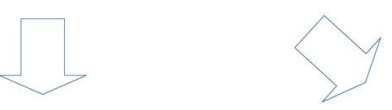

\begin{tabular}{c|c|c}
$\mathcal{D}_{25}^{3}$ & $X_{1}, \ldots, X_{214}$ & $\mathrm{~F} 3$ \\
\hline 1 & $0.5, \ldots, 2.1$ & $\mathrm{Ce}$ \\
2 & $0.2, \ldots, 4.1$ & $\mathrm{De}$ \\
3 & $1.2, \ldots, 4.2$ & $\mathrm{De}$ \\
$\ldots$ & $\ldots$ & $\ldots$ \\
237 & $1.0, \ldots, 2.2$ & $\mathrm{Ce}$
\end{tabular}

\begin{tabular}{c|c|c}
$\mathcal{D}_{25}^{4}$ & $X_{1}, \ldots, X_{214}$ & $\mathrm{~F} 4$ \\
\hline 2 & $0.2, \ldots, 4.1$ & As. \\
3 & $1.2, \ldots, 4.2$ & Ds. \\
$\ldots$ & $\ldots$ & $\ldots$
\end{tabular}

variables in the classification tasks are indicated by the columns of the corresponding data sets (e.g., the predictors for F1 are the morphological parameters $X_{1}-X_{57}$; see $\mathscr{D}_{25}^{1}$ ). Note how label and predictor reliability determines which instances are included in a data set: for example, the first instance in $\mathscr{D}$ (shown in red) is omitted from $\mathscr{D}_{25}^{5}$ because its label reliability is 21 , i.e., it is not above 25 , the label reliability threshold in 55 . Likewise, instance 237 in (shown in orange) is omitted from $\mathscr{D}_{25,21}^{5,1234}$ and $\mathscr{D}_{25,21}^{5,1234, \mathscr{X}}$ because its reliability for F1 is not above 21 . Besides the label reliability thresholds depicted here, many others were considered for each categorization task, e.g., $\mathscr{D}_{24}^{1}$, $\mathscr{D}_{28}^{1}, \mathscr{D}_{30,21}^{5,1234, \mathscr{X}}$, etc

Axonal features $\mathrm{F} 1$ and $\mathrm{F} 2$ refer to the distribution of the axonal arbor relative to the cortical layer and column of the soma, respectively. Cells with the axon predominantly in the soma's cortical layer are intralaminar, whereas the rest are translaminar. Likewise, regarding F2, cells with the axon mainly confined to the soma's cortical column are intracolumnar; the rest are transcolumnar (the column is assumed to have a diameter of $300 \mu \mathrm{m}$ ). Feature $\mathrm{F} 3$ refers to the relative location of axonal and dendritic arbors. Cells with the dendritic arbor mainly located in the center of the axonal arborization are centered whereas the rest are displaced. Feature F4 allows for further distinguishing between translaminar and displaced cells: cells with an axon mainly ascending towards the cortical surface are ascending, cells with an axon mainly descending towards the white matter are descending, whereas the rest are termed both. Feature F5 is the interneuron type. Eight of the ten types can be found in the literature (Peters and Jones 1984), whereas common type 
was introduced by DeFelipe et al. (2013), and other was meant to be chosen when a type missing from the proposal was considered most adequate. Regarding F6, a cell is uncharacterized if its reconstruction does not allow for the characterization according to the remaining features, due to, e.g., insufficient axonal reconstruction; otherwise, a cell is characterized. Supervised models can predict F6 with high accuracy (DeFelipe et al. 2013) so we do not consider this feature in this paper. Figure 2 shows two interneurons characterized according to axonal features F1-F5.

In DeFelipe et al. (2013), expert neuroscientists categorized interneurons by observing images such as those in Fig. 2. In addition, they were told the neuron's cortical layer-along with its approximate thickness-, the cortical area and species of the animal. A gray vertical shadow marked the $300 \mu \mathrm{m}$-wide cortical column, as shown in Fig. 2.

\section{Expert Categorization Reliability}

Each cell was categorized according to every feature by up to 42 experts. To crisply categorize a cell according to an axonal feature $f$, we reduced the vector of experts' choices for $f$ to its mode (majority vote). We used such crisp categorizations as values of the class variable (labels) and of the predictor variables - axonal features F1-F4 were used as predictors of interneuron type. Cells with no unique majority vote for a feature $f$ were discarded from classification tasks that involved $f$, either as the class or as a predictor variable (e.g., a cell without a unique majority vote for F4 was omitted when predicting $\mathrm{F} 4$ and when using $\mathrm{F} 4$ as a predictor of the type; it was used in all other classification tasks, e.g., when predicting F2 from the morphological variables $\left(\mathscr{D}_{25}^{2}\right.$ in Fig. 1)). Furthermore, we formed data subsets with different label reliability thresholds, i.e., such that each instance's label was backed by at least a certain (threshold) number of experts. Thus, a data set $\mathscr{D}_{t}^{f}$, for predicting $f$, was formed of cells with label reliability larger than $t$ for feature $f$, with $t \in\{0, \ldots, 41\}$. When using features F1-F4 as predictors, cells were additionally filtered according to reliability for F1-F4. Thus, a data set $\mathscr{D}_{t, r}^{5,1234}$, for predicting the type with features $\mathrm{F} 1-\mathrm{F} 4$ as predictors, is formed of cells with reliability greater than $r$ for each of the features F1$\mathrm{F} 4$, and reliability greater than $t$ for the interneuron type (the label). A data set $\mathscr{D}_{t, r}^{5,1234, \mathscr{X}}$, with both the morphological parameters and axonal features $\mathrm{F} 1-\mathrm{F} 4$ as predictors of the type, is formed in the same way as $\mathscr{D}_{t, r}^{5,1234}$. When using F1$\mathrm{F} 4$ as predictors, we augmented $\mathrm{F} 4$ with a category called none, to describe the cells which most experts considered as not categorizable according to F4 - these cells would have otherwise been discarded due few experts having categorized them according to $\mathrm{F} 4$, yielding a low reliability for F4. Although this might lead to incorrect categorizationsa cell being translaminar, displaced and neither ascending, descending or both but instead none, in F1, F3, and F4, respectively-such combinations barely appeared in our data (see Fig. 3).

\section{a}

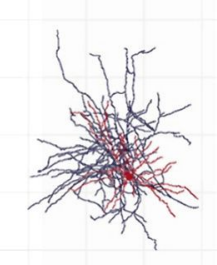

b

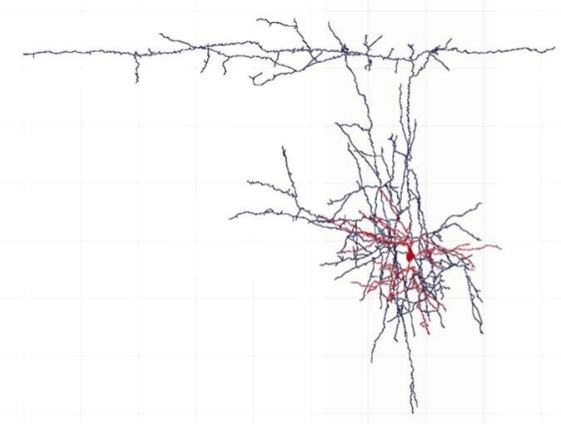

Fig. 2 Examples of interneurons of different types and with different axonal features. a is an intralaminar, intracolumnar, and centered cell, according to 38 (out of 42) experts. Most of its axon (shown in blue) is located within $200 \mu \mathrm{m}$ from the soma (shown in $\mathrm{red}$; the grid lines are established every $100 \mathrm{\mu m}$ ), thus appearing to be in the same layer as the soma; it is within the soma's cortical column (the gray vertical shadows depict a $300 \mu \mathrm{m}$-wide cortical column); and it seems to be centered around the dendritic arbor (also shown in red). Because this cell is not translaminar and displaced, but rather intralaminar and centered, it is not characterizable according to axonal feature F4. According to 24 experts, this is a common basket cell. b is a translaminar, transcolumnar, displaced, and ascending cell according to 38 (out of 42) experts. Unlike (a), this cell's axon reaches over $300 \mu \mathrm{m}$ horizontally above soma (i.e., it seems to extend to another layer); a large portion of its axon is outside of the soma's cortical column; its dendrites are not in the center of the axonal arborization; and its axon is predominantly above the soma. According to 29 experts, this is a Mart inotti cell 


\begin{tabular}{c|c|c|c|c} 
& F1 & F2 & F3 & F4 \\
\hline Expert 1 & translaminar & $\ldots$ & displaced & descending \\
Expert 2 & intralaminar & $\ldots$ & displaced & none \\
Expert 3 & translaminar & $\ldots$ & centered & none \\
\hline Consensus & translaminar & $\ldots$ & displaced & none
\end{tabular}

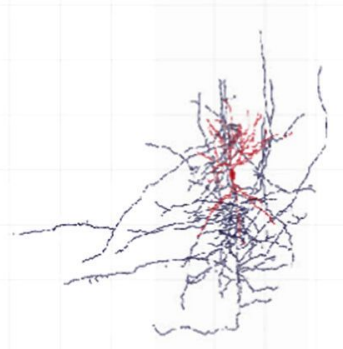

Fig. 3 An example of a theoretically invalid characterization arising due to majority vote categorization-a cell might be categorized as translaminar and displaced in F1 and F3 but neither as ascending, descending, nor both, but instead as none, in F4. The table shows a hypothetical categorization of a cell by three experts. While translaminar and displaced are the modes for F1 and F3, only one expert selected them simultaneously, and therefore only

\section{Morphological Variables}

We used Neurolucida Explorer, the data analysis companion to Neurolucida, to compute 214 parameters of dendritic and axonal morphology using, among others, the following morphological analyses:

- Vertex analysis (described in Sadler and Berry 1983): the count of three types of bifurcations-those with two terminal branches attached $\left(V_{a}\right)$; with one terminal and one bifurcating branch attached $\left(V_{b}\right)$; and with two bifurcating branches attached $\left(V_{c}\right)$.

- Convex hull analysis: various measures of how much space the arbor occupies.

- Sholl analysis: a histogram of intersections of the arbor and a series of concentric spheres centered at the soma. Besides the intersections, we computed histograms of the endings, nodes, and arbor length between two contiguous spheres.

- Fractal analysis (described in Panico and Sterling 1995): box-counting k-dimension - a measure of how well the arbor fills the space.

- Fan-in analysis (Glaser and McMullen 1984): torsion ratio - a measure indicative of any preferred orientation of the arbor.

- Polar histogram (McMullen et al. 1984): a round directional histogram of total arborization length corresponding to an angle interval.

Table 1 shows all the parameters that we computed, grouped by morphological analysis. We applied each analysis, except for 'Dendritic analysis', to both the axon and the dendrites. So, for example, we computed the torsion ratio of the axon and the torsion ratio of the dendrites. In total, we computed 128 axonal and 86 dendritic parameters.

We used all the computed parameters as predictive variables for predicting axonal features F3, F4, and F5. For he/she also categorized the cell according to F4. The rest found that the cell was not characterisable according to F4, which was registered with the value none. none was, therefore, the mode for F4. In our data, there were only three such improperly categorized cells with reliability threshold 21 applied to axonal features F1-F4. One of them is the CT cell (according to 21 experts) shown on the right, characterized as translaminar, intracolumnar, displaced, and none

predicting features $\mathrm{F} 1$ and $\mathrm{F} 2$ we used only the following 57 axonal parameters: total length $\left(\mathbf{B}_{3}\right)$, number of endings $\left(\mathbf{B}_{1}\right)$, mean branch length $\left(\mathbf{B}_{6}\right)$, torsion ratio $\left(\mathbf{F} \mathbf{I}_{1}\right)$, convex hull parameters $\left(\mathbf{C}_{1}-\mathbf{C}_{4}\right)$, Sholl analysis of intersections starting from radius $240 \mu \mathrm{m}\left(\mathbf{S}_{4}-\mathbf{S}_{16}\right)$, and polar histogram $\left(\mathbf{P}_{1}-\mathbf{P}_{36}\right)$.

\section{Discrete Bayesian Network Classifiers}

Let $\mathbf{X}=\left(X_{1}, \ldots, X_{n}\right)$ be the vector of $n$ discrete predictor random variables (or attributes), let $C$ be the (discrete) class random variable, and let $\mathscr{D}=$ $\left\{\left(\mathbf{x}^{(\mathbf{1})}, c^{(1)}\right), \ldots,\left(\mathbf{x}^{(N)}, c^{(N)}\right)\right\}$ be the data sample. A Bayes classifier estimates the probability distribution $p(\mathbf{x}, c)$ from $\mathscr{D}$ and upon classifying it assigns an instance $\mathbf{x}$ to the most probable a posteriori class,

$c^{*}=\underset{c}{\arg \max } p(c \mid \mathbf{x})=\underset{c}{\arg \max } p(\mathbf{x}, c)$.

Unfortunately, $p(\mathbf{x}, c)$ is seldom feasible to estimate because it has exponentially many parameters in $n$. Instead, it is commonly approximated by assuming conditional independences among the predictor variables. Using Bayesian networks (Pearl 1988), even distributions over many variables can be compactly encoded when sufficient conditional independence assumptions are made. Models built on strong independence assumptions, such as the naive Bayes (Minsky 1961), exhibit low variance and are thus especially useful for high dimension small sample data, whereas models that relax these assumptions, such as the tree augmented naive Bayes (Friedman et al. 1997), can outperform them when data suffices to estimate the increased number of parameters. We use examples of both of these types of Bayesian network classifiers; they are described below.

The naive Bayes (Fig. 4a) is a simple but effective classifier (Hand and Yu 2001). It approximates $p(c \mid \mathbf{x})$ by 
Table 1 Axonal and dendritic parameters, grouped by morphological analysis

\begin{tabular}{|c|c|c|}
\hline Analysis & Parameters & Axon Examples \\
\hline \multirow[t]{4}{*}{ Branching } & $\mathbf{B}_{1}-\mathbf{B}_{2}$ Number of endings and bifurcations & $\mathbf{B}_{1}=962.00$ \\
\hline & $\mathbf{B}_{3}-\mathbf{B}_{4}$ Total and mean arbor length & $\mathbf{B}_{3}=41,697.10 \mu \mathrm{m}$ \\
\hline & $\mathbf{B}_{5}-\mathbf{B}_{8}$ Total, mean, median and std. deviation of branch length & $\mathbf{B}_{6}=21.75 \mu \mathrm{m}$ \\
\hline & $\mathbf{B}_{9} \quad$ Highest branch order & $\mathbf{B}_{9}=103.00$ \\
\hline \multirow[t]{2}{*}{ Convex hull } & $\mathbf{C}_{1}-\mathbf{C}_{2}$ Area and perimeter of $2 \mathrm{D}$ convex hull & $\mathbf{C}_{1}=174,185.00 \mu m^{2}$ \\
\hline & $\mathbf{C}_{3}-\mathbf{C}_{4}$ Volume and surface of $3 \mathrm{D}$ convex hull & $\mathbf{C}_{3}=18,864,800.00 \mu m^{3}$ \\
\hline \multirow[t]{5}{*}{ Sholl } & $\begin{array}{l}\text { Spheres of radii }\{60 \mu \mathrm{m}, 2 \times 60 \mu \mathrm{m}, \ldots, R \times 60 \mu \mathrm{m}\} \\
\text { with } R=16 \text { for the axon and } R=5 \text { for the dendrites. }\end{array}$ & \\
\hline & $\mathbf{S}_{1}-\mathbf{S}_{R} \quad$ Intersections with the $R$ spheres & $\mathbf{S}_{1}=57.00$ \\
\hline & $\mathbf{S}_{R+1}-\mathbf{S}_{2 R} \quad$ Endings within the $R$ spheres & $\mathbf{S}_{17}=84.00$ \\
\hline & $\mathbf{S}_{2 R+1}-\mathbf{S}_{3 R}$ Nodes within the $R$ spheres & $\mathbf{S}_{33}=91.00$ \\
\hline & $\mathbf{S}_{3 R+1}-\mathbf{S}_{4 R}$ Arbor length within the $R$ spheres & $\mathbf{S}_{49}=3,528.00 \mu \mathrm{m}$ \\
\hline Fractal & $\mathbf{F}_{1}$ Box-counting k-dimension & $\mathbf{F}_{1}=1.49$ \\
\hline Vertex & $\mathbf{V}_{1}-\mathbf{V}_{4} \quad V_{a}, V_{b}, V_{c}$, and $\frac{V_{a}}{V_{b}}$ & $\mathbf{V}_{1}=225.00$ \\
\hline Branch angle & $\mathbf{B} \mathbf{A}_{1}-\mathbf{B} \mathbf{A}_{9}$ Mean, median, and std. deviation of planar, local and spline bifurcation angles & $\mathbf{B} \mathbf{A}_{1}=1.11 \mathrm{rad}$ \\
\hline Fan-in & $\mathbf{F I}_{1}$ Torsion ratio & $\mathbf{F I}_{1}=1.16$ \\
\hline Polar & 36 angle intervals of width 0.17 radians, starting with [0 rad, $0.17 \mathrm{rad}$ ). & \\
\hline histogram & $\mathbf{P}_{1}-\mathbf{P}_{36}$ Length corresponding to the angle intervals & $\mathbf{P}_{1}=625.40 \mu \mathrm{m}$ \\
\hline \multirow[t]{2}{*}{ Dendritic } & $\mathbf{D}_{1}$ Number of first-order dendrites & Does not apply \\
\hline & $\mathbf{D}_{2}$ Number of bifurcating first-order dendrites & \\
\hline
\end{tabular}

The parameter abbreviations (shown in bold) indicate the number of parameters corresponding to each analysis (e.g., there were nine branching parameters); the exception is Sholl analysis for which there were 20 dendritic and 64 axonal parameters, because more spheres were considered for the axon. The 'dendritic' analysis (lowermost column) applies only to the dendrites. The rightmost column shows examples of applying the analyses to a cell's axon

a

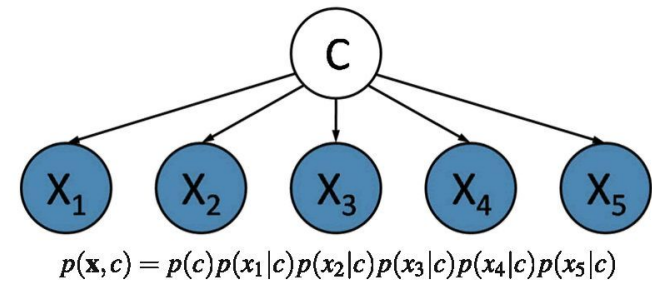

C

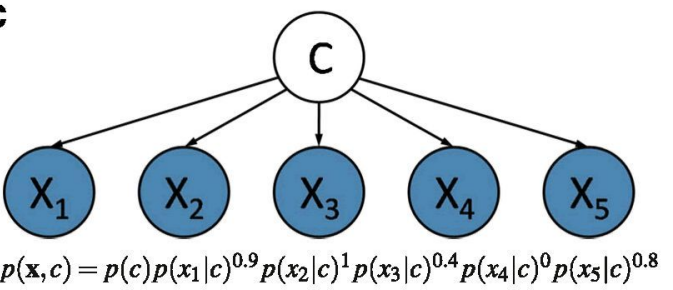

Fig. 4 Examples of the structures of the Bayesian network classifiers used in this paper. Blue nodes denote predictive variables whereas the white node denotes the class variable. The light blue node depicts a domain variable omitted from the model. The formulas are the factorizations of $p(\mathbf{x}, c)$ that follow from the structure above them. a naive Bayes: all predictors are conditionally independent given the class; b selective naive Bayes: predictor $X_{4}$ is omitted from the model; b

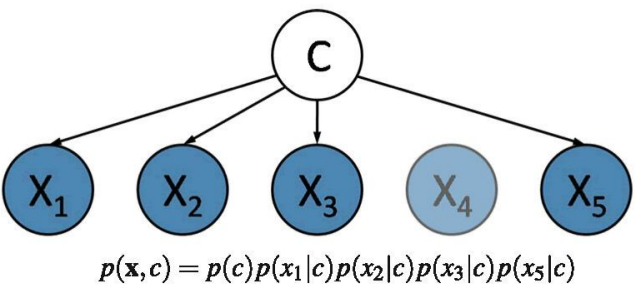

d

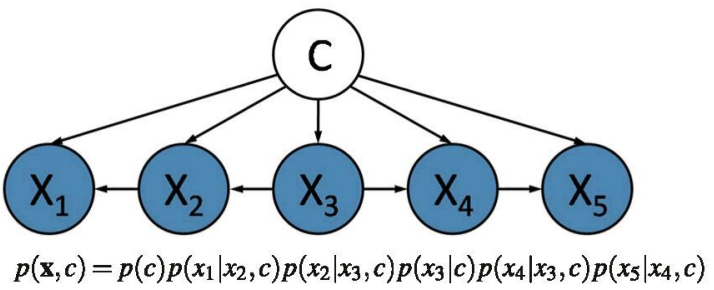

the remaining predictors are independent given the class; $\mathbf{c}$ attribute weighted naive Bayes: factorized like naive Bayes but, due to weights, it yields different class posterior probabilities; note that although $X_{4}$ is included in the model it is irrelevant for computing $p(\mathbf{x}, c)$ because its weight is 0 ; and $\mathbf{d}$ tree augmented naive Bayes, with $X_{3}$ being the root of the augmenting tree 
assuming that the predictors are conditionally independent given the class, i.e.,

$p(c \mid \mathbf{x}) \propto p(c) \prod_{i=1}^{n} p\left(x_{i} \mid c\right)$.

Given its fixed factorization, inducing a naive Bayes amounts to estimating the parameters of the conditional probability distributions.

Feature (predictor) selection can improve the predictive accuracy of naive Bayes (Langley and Sage 1994). Furthermore, parsimonious models can be more cost-efficient and easier to interpret. The forward sequential selection naive

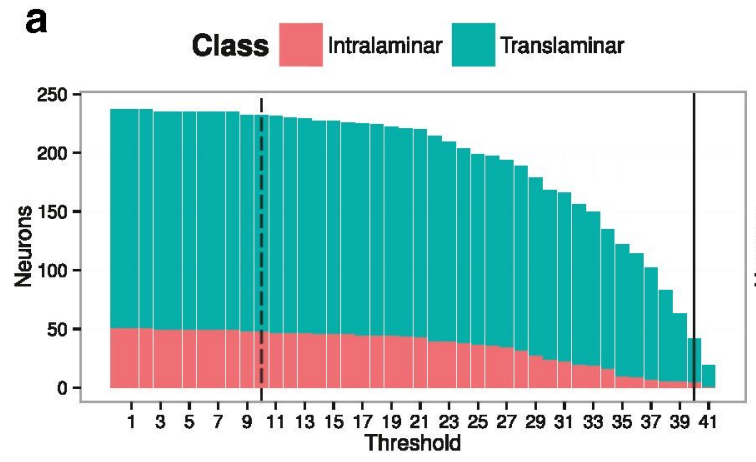

F1

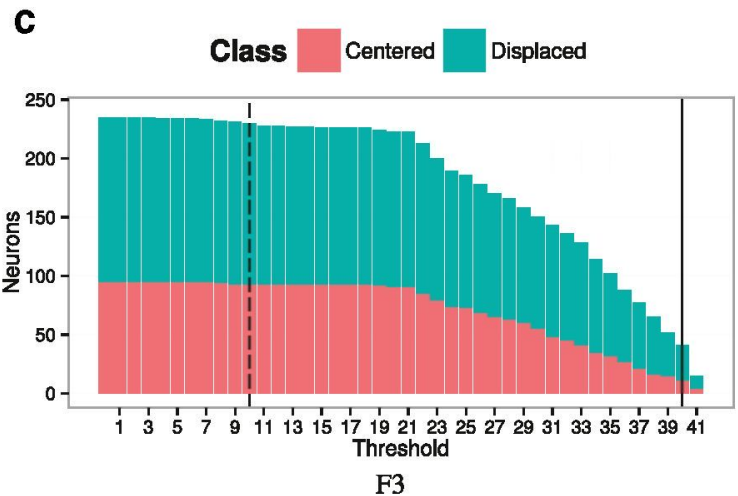

e
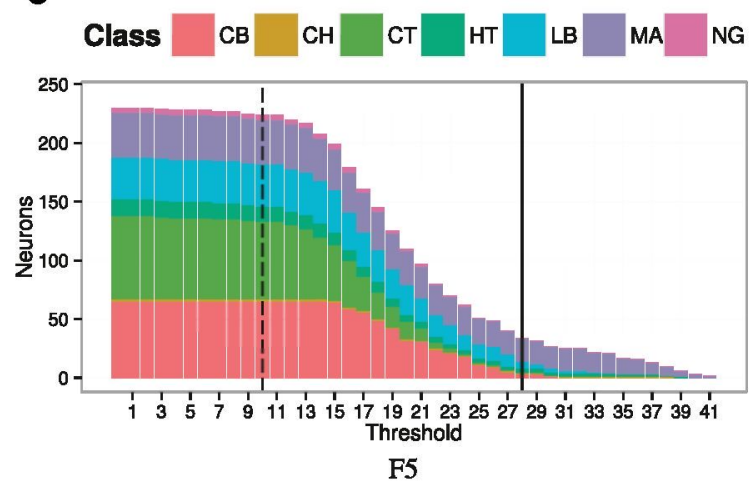

Fig. 5 Number of cells of different classes of F1-F5, versus label reliability threshold. Note that $\mathrm{F} 1$ is unbalanced: there are many more translaminar than intralaminar cells. Regarding F4, there are no both cells above threshold 28 whereas for F5 there are no
Bayes (NB-FSS) algorithm (Langley and Sage 1994) learns a selective naive Bayes (Fig. 4b) with a greedy forward wrapper (i.e., guided by accuracy) search. In other words, it starts from an empty predictor set (a model consisting solely of the class variable) and progressively incorporates predictors as long as they do not degrade predictive accuracy.

A generalization of selective naive Bayes is the weighted naive Bayes (Fig. 4c), given by

$$
p(c \mid \mathbf{x}) \propto p(c) \prod_{i=1}^{n} p\left(x_{i} \mid c\right)^{w_{i}}
$$

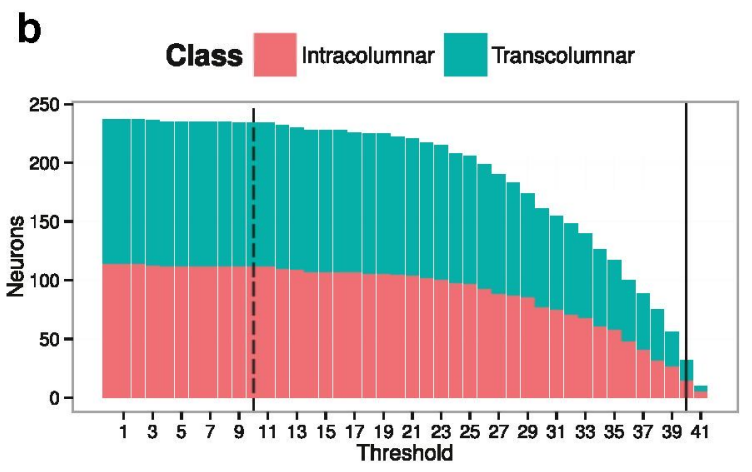

F2
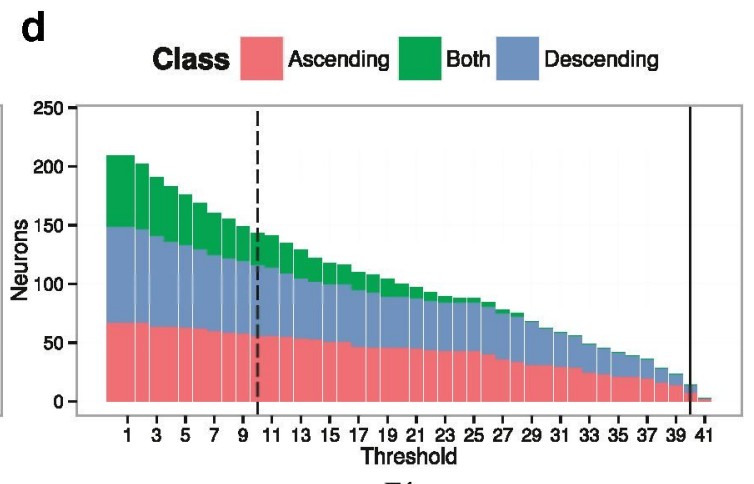

f

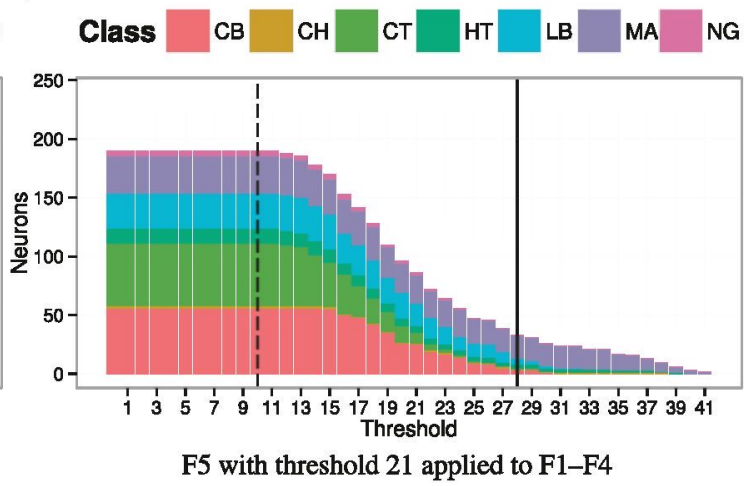

NG cells beyond threshold 24 in both (e) and (f). Solid vertical lines indicate the highest label reliability threshold with no fewer than five instances of at least two classes. Dashed vertical lines indicate the lowest label reliability threshold considered for classification 
a

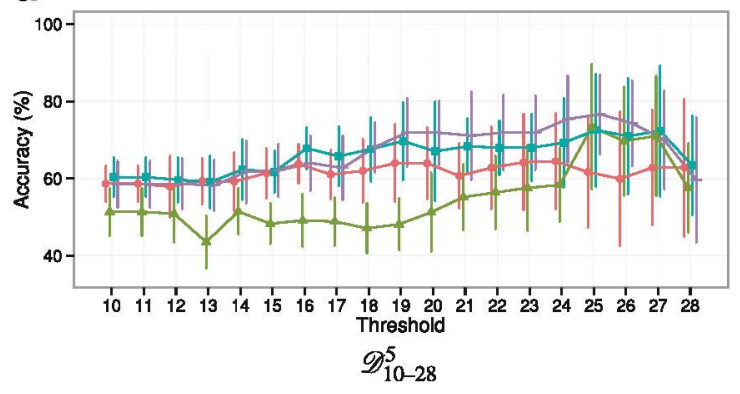

C

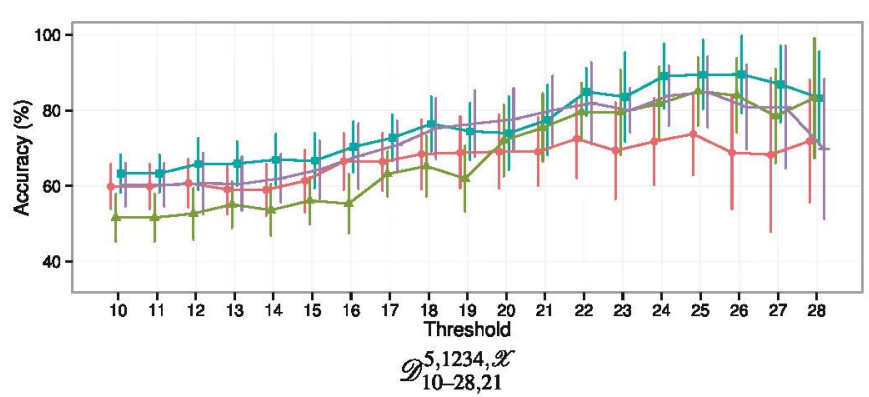

Fig. 6 Interneuron type classification accuracy versus label reliability threshold. a With morphological variables as predictors $\left(\mathscr{D}_{10-28}^{5}\right)$; b with axonal features $\mathrm{F} 1-\mathrm{F} 4$ as predictors $\left(D_{10-28,21}^{5,1234}\right)$; and $\mathbf{c}$ with axonal features $\mathrm{F} 1-\mathrm{F} 4$ and morphological variables as predictors $\left(\mathscr{D}_{10-28,21}^{5,1234, \mathscr{X}}\right)$. For (b) and (c), values of F1-F4 were obtained with

with $w_{i} \in[0,1]$ (predictor selection amounts to using weights $w_{i} \in\{0,1\}$; Zaidi et al. 2013). The attribute weighted naive Bayes classifier (AWNB; Hall 2007) weights a predictor $X_{i}$ in inverse proportion to $X_{i}$ 's dependence on other features; this dependence is estimated as

a

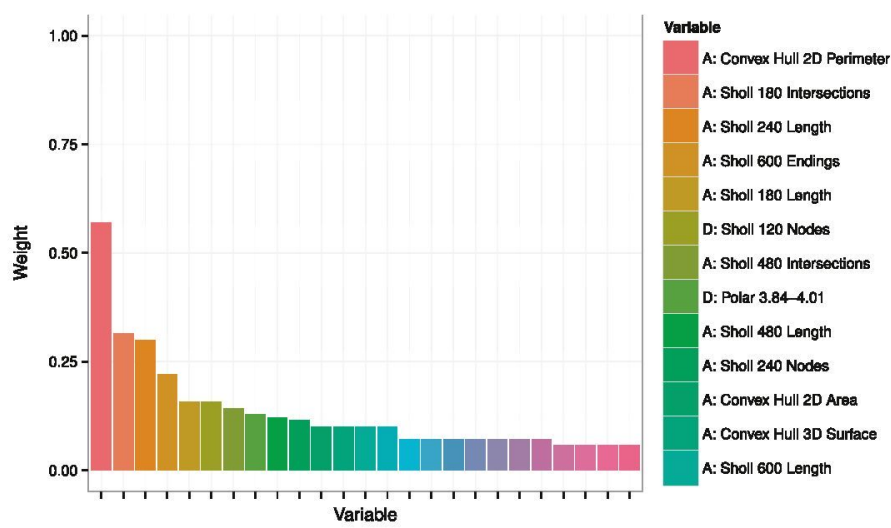

Fig. 7 AWNB weights for predictor variables at a: $\mathscr{P}_{25}^{5}$ and $\mathbf{b}$ : $\mathscr{D}_{26,21}^{5,1234, \mathscr{X}}$. Only variables with weights greater than zero are shown. 'A:' denotes an axonal variable whereas a 'D:' denotes a dendritic variable. The numbers in Sholl variables refer to distances of spheric rings from soma, in $\mu \mathrm{m}$, e.g., 'A: Sholl 600 Length' is the axonal arborization length within the spheric ring at $540 \mu \mathrm{m}-600 \mu \mathrm{m}$ from soma. The b

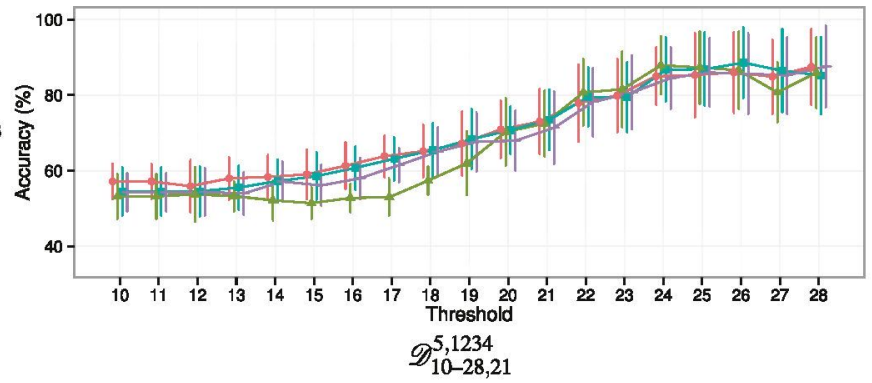

d

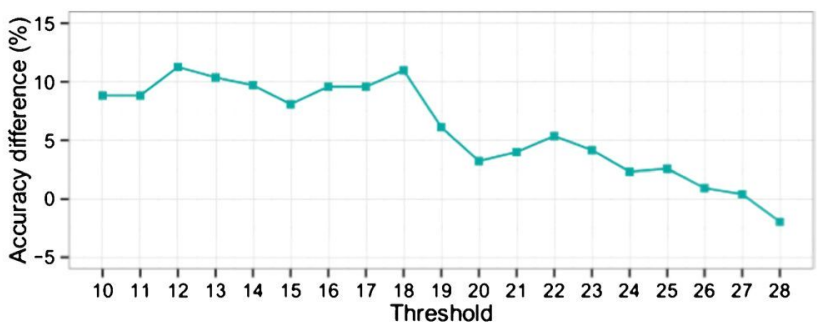

AWNB at (c) - AWNB at (b)

a label reliability threshold 21 , the used data thus being a subset of data used in (a). b and $\mathbf{c}$ were produced by identical cross-validation partitions (i.e., no differences between them due to chance). d plots the accuracy of AWNB at $\mathscr{D}_{10-28,21}^{5,1234, \mathscr{X}}$ minus its accuracy at $\mathscr{D}_{10-28,21}^{5,1234}$. Error bars in (a), (b), and (c) show the standard deviation of accuracy from five runs of five-fold cross-validation

$\frac{1}{\sqrt{d_{i}}}$, where $d_{i}$ is the minimum depth at which $X_{i}$ is tested in an unpruned decision tree $\left(\frac{1}{\sqrt{d_{i}}}=0\right.$ is assumed if $X_{i}$ is not in the tree). To stabilize the estimate, various trees are constructed using bagging (Breiman 1996) and the estimates are averaged across the ensemble.

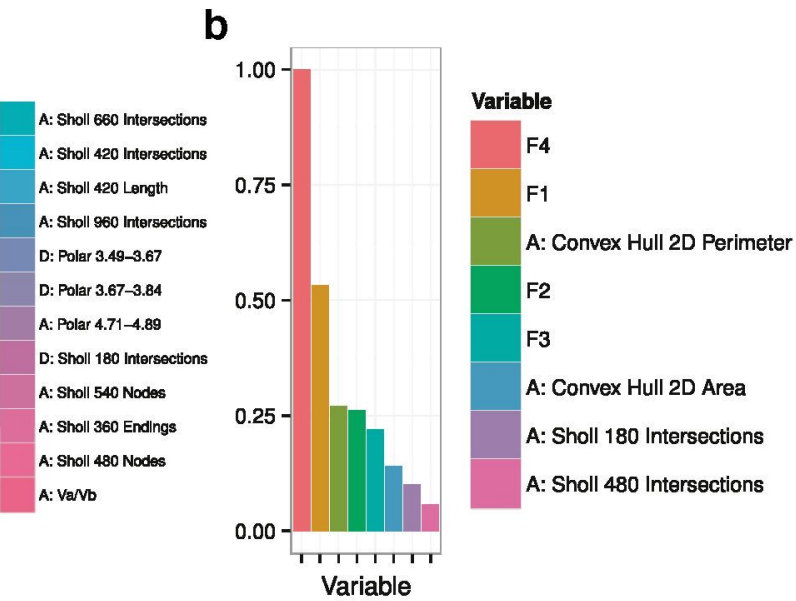

numbers in the polar histogram variables refer to radian intervals, e.g., 'A: Polar 4.71-4.89' refers to axonal arborization length corresponding to the angle between 4.71 and 4.89 radians. The weights were computed by learning an AWNB model from the full data set, after selecting the 100 variables with the highest mutual information with the class variable 
Table 2 Top: NB-FSS' confusion matrix for $\mathscr{Q}_{25}^{5}$ (i.e., for predicting feature F5 at threshold 25; Fig. 1 explains this notation)

\begin{tabular}{|c|c|c|c|c|c|c|}
\hline & $\mathrm{CB}$ & $\mathrm{CH}$ & $\mathrm{CT}$ & $\mathrm{HT}$ & $\mathrm{LB}$ & $\mathrm{MA}$ \\
\hline $\mathrm{CB}$ & 10 & 1 & & & 2 & 1 \\
\hline \multicolumn{7}{|l|}{$\mathrm{CH}$} \\
\hline \multicolumn{7}{|l|}{$\mathrm{CT}$} \\
\hline \multicolumn{7}{|l|}{$\mathrm{HT}$} \\
\hline $\mathrm{LB}$ & & & & & 9 & 2 \\
\hline $\mathrm{MA}$ & 1 & & 1 & 4 & 1 & 19 \\
\hline Sensitivity & 0.91 & 0.00 & 0.00 & 0.00 & 0.75 & 0.86 \\
\hline Specificity & 0.90 & 1.00 & 1.00 & 1.00 & 0.95 & 0.76 \\
\hline
\end{tabular}

Columns denote the true classes whereas rows denote the predicted classes. Zeros were omitted. Bottom: NB-FSS' sensitivity and specificity per class. All values in the table were computed from a single run of stratified five-fold cross-validation and might not, therefore, exactly match the accuracy reported in Fig. 6a (yielded by five runs)

The tree augmented naive Bayes (Fig. 4d) alleviates some of naive Bayes' conditional independence assumptions. All predictors except for one-the root of the augmenting tree-are conditioned on a single other predictor, yielding

$p(c \mid \mathbf{x}) \propto p(c) p\left(x_{r} \mid c\right) \prod_{i \neq r} p\left(x_{i} \mid x_{j(i)}, c\right)$,

where $X_{r}$ is the tree root and $X_{j(i)}$ is the predictor that $X_{i}$ is conditioned on. This classifier is efficiently learned by maximizing the likelihood of the resulting structure.

In summary, we used four Bayesian network classifiers of different complexities (i.e., conditional independence assumptions) and predictor selection and weighting techniques. We will refer to their learning algorithms with the following abbreviations: naive Bayes$\mathrm{NB}$; forward sequential selection naive Bayes-NB-FSS; attribute-weighted naive Bayes-AWNB; and tree augmented Bayes-TAN.

\section{Discretization and Dimensionality Reduction}

Before classifier induction, we converted all numeric variables (i.e. the morphological parameters) to categorical ones. This process, known as discretization (Yang et al. 2010), often yields more accurate naive Bayes classifiers than when assumptions such as that of normality are made about the underlying probability distributions (Dougherty et al. 1995). We used the equal-frequency discretization technique and determined the number of intervals as a function of data set size, following the weighted proportional k-interval discretization (WPKID) method (Yang and Webb 2003). The discretization process did not bias accuracy estimates as it was guided only by training data (within a cross-validation scheme): the test data were mapped, upon classification, to the intervals learned from training data.

The number of predictor variables (up to 218) was possibly too high for NB and TAN to perform well, as they do not perform predictor selection. Thus, after discretizing the training data and before inducing the classifiers (i.e., on the training set within a cross-validation scheme), we reduced the predictor set to the 100 variables with the highest mutual information with the class variable. ${ }^{2}$ Since predictors were selected from the training set alone, this did not bias the cross-validated accuracy estimates (Smialowski et al. 2010).

\section{Empirical Setup}

\section{Label Reliability Thresholds}

The number of cells, naturally, decreased with higher label reliability. We only considered label reliability thresholds with no fewer than five instances of at least two classes, which provided the upper bounds for the reliability thresholds used: the bound was 40 for axonal features F1-F4 (see Fig. 5a-d), and 28 for F5 (Fig. 5e). The lower bound in all classification tasks was ten, roughly corresponding to one quarter of the experts.

There were seven interneuron types up to threshold 24; no NG cells remained on higher thresholds. Regardless of the threshold there were fewer than five $\mathrm{CH}, \mathrm{HT}$, and NG cells, making these types especially hard to identify. Regarding F4, no both cells remained above threshold 28 . The

\footnotetext{
${ }^{2}$ This was not applied in classification tasks with less than 100 predictors, e.g., when predicting the interneuron type with only F1-F4 as predictor variables.
} 
Table 3 Top: TAN's confusion matrix for $\mathscr{D}_{25}^{5}$

\begin{tabular}{|c|c|c|c|c|c|c|}
\hline & $\mathrm{CB}$ & $\mathrm{CH}$ & $\mathrm{CT}$ & $\mathrm{HT}$ & $\mathrm{LB}$ & MA \\
\hline $\mathrm{CB}$ & 10 & 1 & & & 1 & \\
\hline \multicolumn{7}{|l|}{$\mathrm{CH}$} \\
\hline \multicolumn{7}{|l|}{$\mathrm{CT}$} \\
\hline HT & & & & 3 & & \\
\hline $\mathrm{LB}$ & & & & & 8 & 3 \\
\hline MA & 1 & & 1 & 1 & 3 & 19 \\
\hline Sensitivity & 0.91 & 0.00 & 0.00 & 0.75 & 0.67 & 0.86 \\
\hline Specificity & 0.95 & 1.00 & 1.00 & 1.00 & 0.92 & 0.79 \\
\hline
\end{tabular}

Columns denote the true classes whereas rows denote the predicted classes. Zeros were omitted. Bottom: TAN's sensitivity and specificity per class. All values in the table were computed from a single run of stratified five-fold cross-validation and might not, therefore, exactly match the accuracy reported in Fig. 6 a (yielded by five runs)

Table 4 Top: AWNB's confusion matrix for $\mathscr{D}_{26,21}^{5,1234}$

\begin{tabular}{|c|c|c|c|c|c|c|}
\hline & $\mathrm{CB}$ & $\mathrm{CH}$ & $\mathrm{CT}$ & $\mathrm{HT}$ & $\mathrm{LB}$ & $\mathrm{MA}$ \\
\hline $\mathrm{CB}$ & 6 & & 1 & & 1 & \\
\hline \multicolumn{7}{|l|}{$\mathrm{CH}$} \\
\hline \multicolumn{7}{|l|}{ CT } \\
\hline HT & & 1 & & 4 & & \\
\hline LB & 2 & & & & 9 & \\
\hline MA & & & & & 1 & 21 \\
\hline Sensitivity & 0.75 & 0.00 & 0.00 & 1.00 & 0.82 & 1.00 \\
\hline Specificity & 0.95 & 1.00 & 1.00 & 0.98 & 0.94 & 0.96 \\
\hline
\end{tabular}

Columns denote the true classes whereas rows denote the predicted classes. Zeros were omitted. Bottom: AWNB's sensitivity and specificity per class. All values in the table were computed from a single run of stratified five-fold cross-validation and might not, therefore, exactly match the accuracy reported in Fig. $6 \mathrm{~b}$ (obtained by five runs)

Fig. 8 AWNB predictor weights for predicting F5 from axonal features F1-F4 versus label reliability threshold. Note that the order of importance of the features is constant across the thresholds: most weight is given to F4, least to F3, with F2 and $\mathrm{F} 1$ in between

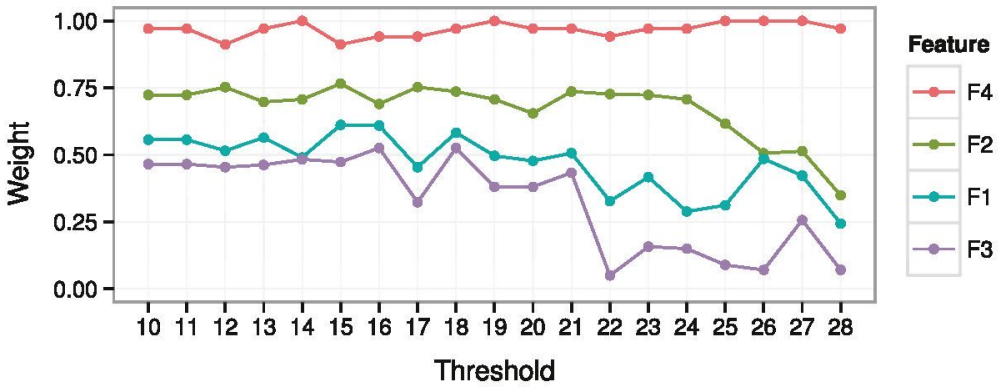




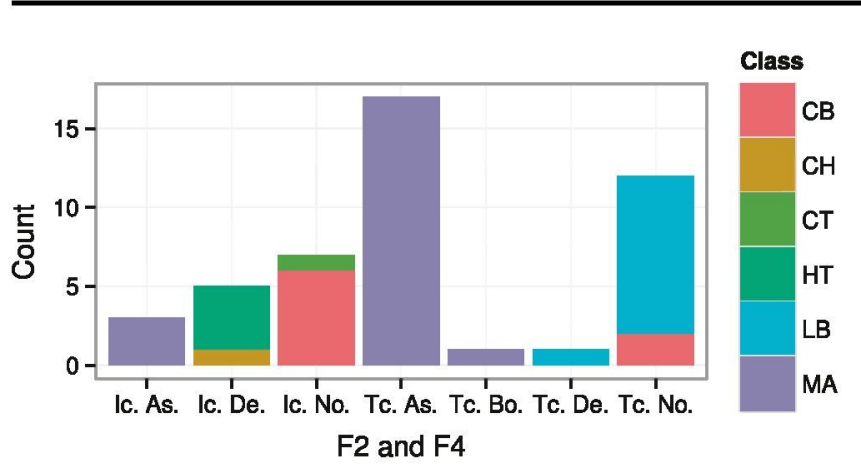

Fig. 9 Interneuron type (colours) versus combination of F2 and F4 values at threshold 26 for interneuron type and threshold 21 for F1-F4 $\left(\mathscr{D}_{26,21}^{5,1234}\right) . \mathrm{F} 2$ and F4 combined could discriminate between $\mathrm{CB}, \mathrm{HT}$, $\mathrm{LB}$, and MA and cells rather precisely (e.g., all transcolumnar and ascending cells were MA). Feature F4 alone separated MA from HT: all HT were descending whereas MA were either ascending or both, whereas $\mathrm{F} 2$ largely separated $\mathrm{CB}$ from $\mathrm{LB}$ cells: all $\mathrm{LB}$ were transcolumnar while most $\mathrm{CB}$ were intracolumnar. Abbreviations: Ic. = intracolumnar; Tc. = transcolumnar; As. = ascending; De. = descending; Bo. = both; and No. = none

predictions of $\mathrm{F} 1, \mathrm{~F} 2$, and $\mathrm{F} 3$ were binary tasks regardless of label reliability threshold, i.e., the same classes were present at all thresholds considered.

When using F1-F4 as predictors of the interneuron type (F5) we fixed the reliability threshold for F1-F4 to $21-$ corresponding to $50 \%$ of the experts-while considering all thresholds from 10 to 28 for F5 (see Fig. 5f), following the above-described criteria.

\section{Classifier Parametrization and Accuracy Estimation}

For NB-FSS we used resubstitution accuracy as the objective function; we halted its search process when new accuracy improved current accuracy by no more than $10 \%$ (i.e., $\left.a c c_{\text {new }}-a c c_{\text {current }} \leq 10, a c c \in[0,100]\right)$. For the AWNB classifier, we built classification trees from 10 bootstrap samples half the size of the data set $\left(\frac{N}{2}\right)$. We estimated the parameters of the Bayesian networks with Laplace correction for maximum likelihood. We estimated predictive accuracy of the classifiers with five repetitions of five-fold stratified cross-validation.

\section{Software}

The Bayesian network classifiers used are implemented in the bnclassify (Mihaljevic et al. 2013) package for the R (R Core Team 2012) statistical software environment. We used weka (Hall et al. 2009) for discretization (through the RWeka (Hornik et al. 2009) interface for R) and the caret R package (Kuhn et al. 2013) for cross-validation estimation of accuracy.

\section{Results}

Predicting Interneuron Type

From Morphological Variables

In general, accuracy improved with label reliability (see Fig. 6a). Best accuracy-76.63\%-was achieved by TAN at label reliability threshold $25\left(\mathscr{D}_{25}^{5}\right)$. While a TAN model incorporates all predictive variables, ${ }^{3}$ AWNB and NB-FSS achieved comparable accuracy, at this threshold, with few variables: AWNB with 24 (19 axonal and five dendritic) and NB-FSS with two axonal variables: the 2D convex hull perimeter $\left(\mathbf{C}_{2}\right)$ and Sholl intersections at $180 \mu \mathrm{m}$ from soma $\left(\mathbf{S}_{3}\right)$; these were also the most relevant variables according to AWNB-see Fig. 7a. At thresholds 25-27 NB-FSS was very accurate by using these two variables alone, indicating that they suffice for discriminating among $C B$, $L B$, and $M A$ cells, which are the interneuron types that NB-FSS was able identify at these thresholds (see Table 2). Unlike NB-FSS, TAN also managed to identify $\mathrm{HT}$ cells at threshold 25 , thus accurately discriminating among CB, HT, LB, and MA cells (see Table 3).

\section{From Axonal Features F1-F4}

In general, accuracy improved with label reliability (see Fig. 6b) and NB, AWNB, and TAN were similarly accurate at all thresholds. The best accuracy- $88.58 \%$-was achieved by AWNB at threshold $26\left(\mathscr{D}_{26,21}^{5,1234}\right)$. All classifiers could accurately discriminate between reliable examples of the $\mathrm{CB}, \mathrm{HT}$, LB, and MA types (see Table 4 for AWNB). Not only were they similarly accurate but they actually classified in a similar way-for example, TAN and NB-FSS had identical confusion matrices at threshold 26. Prediction was more accurate than with morphological predictors alone (note that, although different, the data sets from the two settings were actually similar at high reliability thresholds, see Fig. 5e and f).

F4 seemed to be the most useful axonal feature for predicting the interneuron type. Regardless of label reliability, AWNB always assigned most importance to F4, then to F2, and least to F1 and F3 (see Fig. 8). Accordingly, the NB-FSS classifier selected F4, while omitting F1 and F3, at all thresholds; when it selected F2 along with F4 at thresholds 20-27-it was more or similarly accurate as the remaining classifiers. Indeed, features F4 and F2 alone could separate reliable examples of the $\mathrm{CB}, \mathrm{HT}$, LB, and MA types (see Fig. 9). The omission of F1 and $\mathrm{F} 3$, in favour of F4, by NB-FSS, and their lower importance in $\mathrm{AWNB}$, is reasonable since $\mathrm{F} 4$ by definition carries

\footnotetext{
${ }^{3}$ The 100 variables that were selected previous to classifier induction.
} 
a

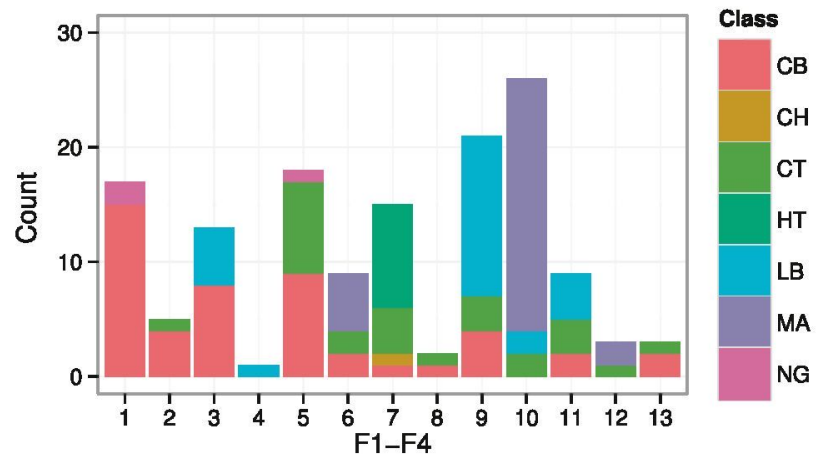

b

\begin{tabular}{cccccccc}
\hline & CB & CH & CT & HT & LB & MA & NG \\
\hline CB & 39 & & 11 & & 5 & & 3 \\
CH & & & & & & & \\
CT & & & & & & & \\
HT & 1 & 1 & 4 & 9 & & & \\
LB & 6 & & 6 & & 19 & & \\
MA & 2 & & 5 & & 2 & 29 & \\
NG & \multicolumn{7}{c}{ Accuracy: $67.60 \%$}
\end{tabular}

C

\begin{tabular}{cccccccc}
\hline & CB & CH & CT & HT & LB & MA & NG \\
\hline CB & 36 & & 9 & & 6 & & 3 \\
CH & & & & & & & \\
CT & 1 & & 1 & & 3 & & \\
HT & 1 & 1 & 4 & 9 & & & \\
LB & 7 & & 6 & & 15 & & \\
MA & 3 & & 6 & & 2 & 29 & \\
NG & \multicolumn{7}{c}{ Accuracy: $63.38 \%$}
\end{tabular}

Fig. 10 A combination of features F1-F4 does not clearly identify the interneuron type at a low reliability threshold. a plots the interneuron type against the combinations of features $\mathrm{F} 1-\mathrm{F} 4$ at $\mathscr{D}_{17,21}^{5,1234}$; b is the confusion matrix of an 'ideal' classifier which would assign every combination of F1-F4 values in (a) to its most common class. Thus, for example, the five LB cells corresponding to combination 3 (blue part of the third bar in (a)) would be assigned to $\mathrm{CB}$ (salmon-colored part of the same bar), since $C B$ cells are predominant in combination 3 . The confusion matrix of AWNB is shown in (c) whereas the difference between the 'ideal' confusion matrix and that of AWNB, (b)-(c), is shown in (d). AWNB was only slightly worse that the 'ideal' classifier: it misclassified three $\mathrm{CB}$ and four LB cells more than 'ideal' classifier (shown in red in (d)) but correctly classified one CT cell more (shown in blue). The latter is possible due to random permutations in crossvalidation. The columns in confusion matrices denote the true classes whereas rows denote the predicted classes. Zeros were omitted.

information about $\mathrm{F} 1$ and $\mathrm{F} 3$ - a cell that is none in F4 is not, by definition, translaminar and displaced (in F1 and F3, respectively), whereas a cell with a different F4 value (ascending, descending, or both) is translaminar and displaced (in F1 and F3, respectively). This redundancy of F4 on the one hand and $\mathrm{F} 1$ and F3 on the other might suggest that predictor weighting is an adequate approach.

Nevertheless, not even the combination of the four highlevel axonal features is expressive enough to separate the types well at low reliability thresholds. Many cells had identical axonal features $F 1-F 4$ but nevertheless belonged to different types; since a single combination of features can only be assigned to one type, many cells cannot be correctly classified. Poor accuracies at low thresholds are partially
The confusion matrix of the AWNB was obtained from a single run of cross-validation. F1-F4 combinations: 1 = (intralaminar, intracolumnar, centered, none); 2 = (intralaminar, intracolumnar, displaced, none); 3 = (intralaminar, transcolumnar, centered, none); 4 = (intralaminar, transcolumnar, displaced, none); 5 = (translaminar, intracolumnar, centered, none); $6=$ (translaminar, intracolumnar, displaced, ascending); 7 = (translaminar, intracolumnar, displaced, descending); 8 = (translaminar, transcolumnar, displaced, both); 9 = (translaminar, transcolumnar, centered, none); $10=$ (translaminar, transcolumnar, displaced, ascending); 11 = (translaminar, transcolumnar, displaced, descending); 12 = (translaminar, transcolumnar, displaced, both); and 13 = (trans laminar, transcolumnar, displaced, none)

due to this limited expressiveness; in fact, they are close to the accuracies achievable by assigning each instance to its majority class (see Fig. 10). This suggests that a richer predictor space (i.e., beyond F1-F4) might be necessary to better discriminate among interneuron types at low thresholds. For this purpose, we augmented the predictor set with the 214 morphological variables; the obtained results are presented in the following section.

\section{From Morphological Variables and Axonal Features F1-F4}

Using the morphological variables together with the highlevel axonal features improved AWNB's accuracy at all thresholds except for 28 (see Fig. 6c and d). AWNB achieved the highest overall accuracy (considering all 
a

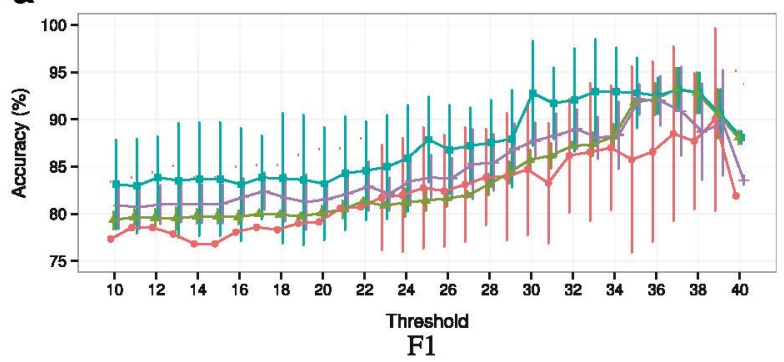

F1

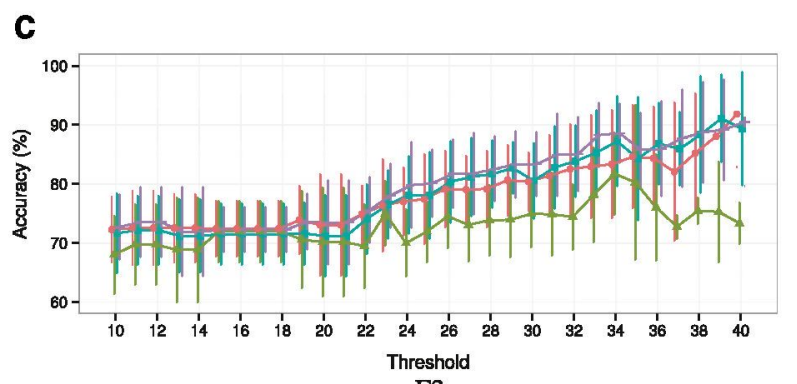

F3
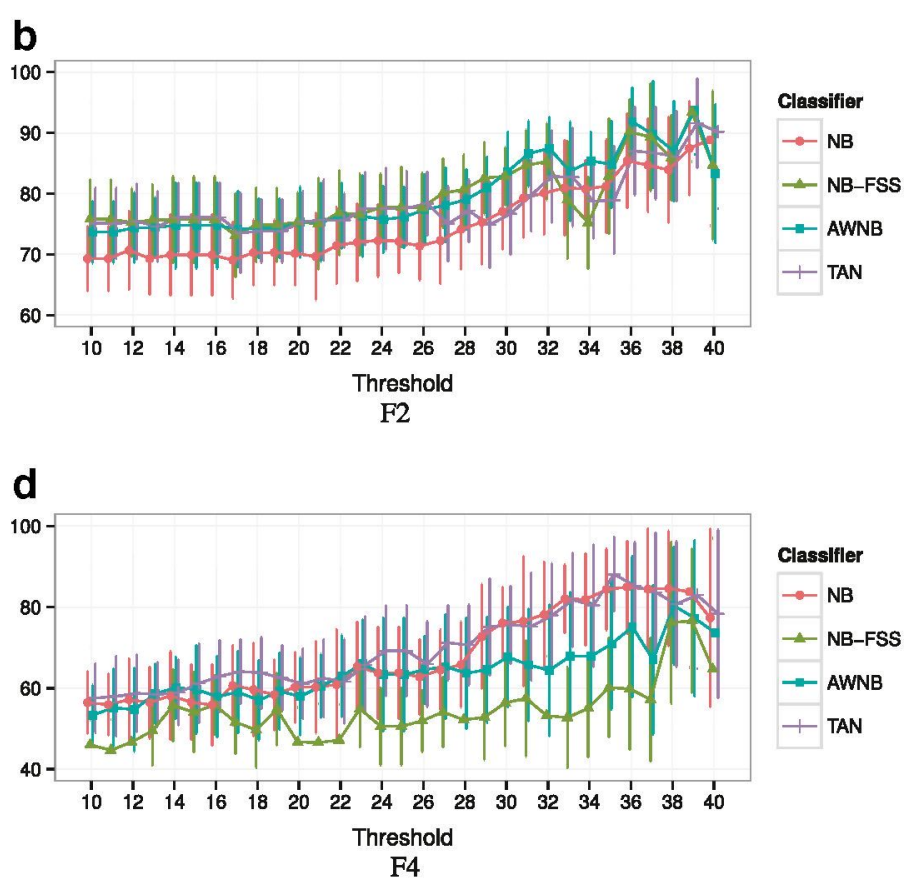

Fig. 11 Classification accuracy for axonal features F1-F4 ((a)-(d), respectively) versus label reliability threshold. Error bars show standard deviation of accuracy from five runs of five-fold cross-validation

three predictor sets) for predicting the interneuron type$89.52 \%$-at threshold $26\left(\mathscr{D}_{26,21}^{5,1234, \mathscr{X}}\right)$. At this threshold, it assigned non-zero weights to eight predictors: the features F1-F4 and four morphological parameters of the axon (see Fig. 7b), with most weight assigned to F4. These morphological parameters were also used by AWNB in 'From Morphological Variables' (see Fig. 7a). In both settings most weight was assigned to the $2 \mathrm{D}$ convex hull perimeter $\left(\mathbf{C}_{2}\right)$.

Augmenting the predictor set with morphological variables also improved the accuracies of TAN and NB-which do not prune the predictor set-at low thresholds (up to thresholds 22 and 19 for TAN and NB, respectively; see Fig. $6 c$ ). This seems to confirm that poor accuracies at low thresholds in the previous setting were partially due to the limited expressiveness of features F1-F4. NB and TAN performed worse at high thresholds - where features F2 and F4 suffice to discriminate among the types-, possibly because axonal features F1-F4 were dominated by the many morphological variables, only some of which seem to be useful for class prediction.

\section{Predicting Axonal Features F1-F4}

The highest accuracy for predicting the axonal feature F1 $-93.19 \%$ - was achieved by the AWNB and NB-FSS classifiers at threshold $37\left(\mathscr{D}_{37}^{1}\right.$; see Fig. 11a). These classifiers, however, assigned all cells to the predominant translaminar class (at threshold 37 there were 95 translaminar and 7 intralaminar cells). NB was best at correctly identifying intralaminar cells but had a lower accuracy that the remaining classifiers $(88.43 \%$; see Table 5).

Regarding the prediction of the axonal feature F2, the highest accuracy-93.75\%-was achieved by AWNB at label reliability threshold $39\left(\mathscr{D}_{39}^{2}\right.$; see Fig. 11b). It was similarly good at identifying both categories of interneurons $(0.95$ sensitivity and 0.91 specificity, with intracolumnar being the positive class). Generally, AWNB was most accurate at classifying reliably labeled cells (it was the best at thresholds 17 and 31-39) whereas $\mathrm{NB}$ was most often the least accurate, indicating that the predictor variables were redundant to some degree. AWNB used ten variables at threshold 39 (see Fig. 12a), with most weight assigned to convex hull $2 \mathrm{D}$ area $\left(\mathbf{C}_{1}\right)$, which was the only variable selected by NB-FSS - similarly accurate

Table 5 Sensitivity, specificity and accuracy of the different classifiers at $\mathscr{D}_{37}^{1}$. intralaminar is considered as the positive class

\begin{tabular}{llll}
\hline & Sensitivity & Specificity & Accuracy \\
\hline NB & 1 & 0.88 & $88.43 \%$ \\
NB-FSS & 0 & 1 & $93.19 \%$ \\
AWNB & 0 & 1 & $93.19 \%$ \\
TAN & 0.11 & 0.97 & $90.78 \%$ \\
\hline
\end{tabular}




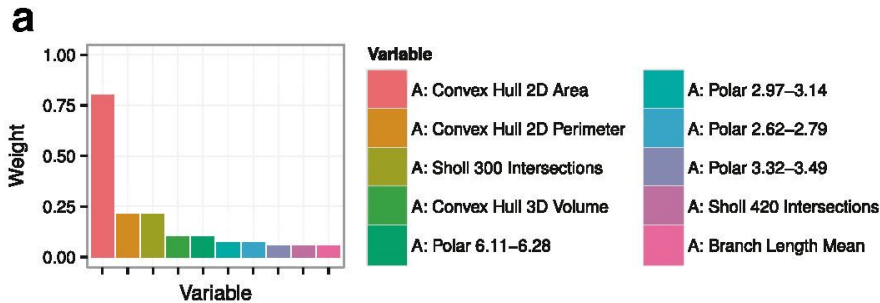

Fig. 12 AWNB weights for predictor variables at a: $\mathscr{D}_{39}^{2}$ and b: $\mathscr{D}_{40}^{3}$. Only variables with weights greater than zero are shown. 'A:' denotes an axonal variable whereas a ' $D$ :' denotes a dendritic variable. The numbers in Sholl variables refer to distances of spheric rings from soma, in $\mu \mathrm{m}$, e.g., 'A: Sholl 600 Length' is the axonal arborization length within the spheric ring at $540 \mu \mathrm{m}-600 \mu \mathrm{m}$ from soma. The

as $\mathrm{AWNB}$ - at this threshold. Indeed, NB-FSS selected a single convex hull variable at each threshold-either $\mathbf{C}_{1}$, $\mathbf{C}_{3}$, or $\mathbf{C}_{4}$-and it was nonetheless relatively accurate (scoring within $2 \%$ of the highest accuracy at all but three thresholds).

Regarding the prediction of $\mathrm{F}$, the highest accuracy$91.83 \%$ - was achieved by NB at label reliability threshold $40\left(\mathscr{D}_{40}^{3}\right.$; see Fig. 11c). NB was slightly more accurate, at this threshold, at identifying the more numerous displaced cells ( 0.87 sensitivity and 0.93 specificity, with centered being the positive class). Although F4 refers to the relative distribution of axonal and dendritic arbors, AWNB was similarly accurate as NB at this threshold by using only eight axonal variables (see Fig. 12b). Although AWNB selected no more than 48 variables at a single threshold (with the number of variables inversely proportional to label reliability), it achieved similar accuracy as NB at most thresholds, which might suggest that only a subset of variables is useful for predicting the axonal feature F3.

The highest accuracy for predicting the axonal feature F4-88.10\%-was achieved by TAN at threshold 35 $\left(\mathscr{V}_{35}^{4}\right.$; see Fig. 11d). At this threshold there were no both cells and the classifiers thus had to distinguish between ascending and descending cells alone. TAN was equally good at identifying both classes $(0.88$ sensitivity and specificity). When distinguishing between ascending and descending cells, NB and TAN outperformed the classifiers that prune the predictor set-AWNB and NBFSS - (see Fig. 11d), which may suggest that many of the morphological variables used are useful for distinguishing among these two categories.

\section{Conclusions}

We used supervised classifiers based on Bayesian networks to automatically categorize cortical GABAergic

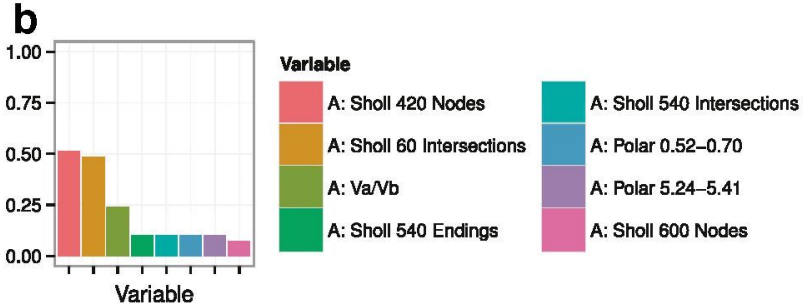

numbers in the polar histogram variables refer to radian intervals, e.g., 'A: Polar 4.71-4.89' refers to axonal arborization length corresponding to the angle between 4.71 and 4.89 radians. The weights were computed by learning an AWNB model from the full data set, after selecting the 100 variables with the highest mutual information with the class variable

interneurons according to their type-as commonly used in the literature - and four features of axonal arborization, called F1, F2, F3, and F4. We trained the classifiers with the categorization of 237 interneurons according to the mentioned features and the interneuron type provided by 42 expert neuroscientists. We used up to 214 morphological parameters as predictor variables. Also, we used the axonal features F1-F4 as predictors of interneuron type. Due to little inter-expert agreement on the categorization of some cells, we separately analyzed data subsets with different expert categorization reliability thresholds.

We found that the interneurons that were categorized with more inter-expert agreement were more accurately classified by our models. The models accurately distinguished between reliable examples of the common basket, horse-tail, large basket and Martinotti interneuron types. Analyzing the Bayesian network classifiers, we identified two axonal variables - the convex hull $2 \mathrm{D}$ perimeter and number of branches at $180 \mu \mathrm{m}$ from somaand the axonal features $\mathrm{F} 1-\mathrm{F} 4$ as especially useful for discriminating among these interneuron types. Indeed, axonal features F2 and F4 alone were able to accurately separate reliable examples of these types. Besides the interneuron type, we were also able to accurately categorize interneurons according to the axonal features F1-F4.

Since we show that axonal features F1-F4 can be accurately predicted, it might be possible to avoid recurring to experts for future neuron labellings according to these features and instead use the values provided by the models. For this purpose, it would be useful to predict the axonal features simultaneously,-using, e.g., multidimensional Bayesian network classifiers (Bielza et al. 2011) —instead of separately, as in the present study. Furthermore, using the predicted values of $\mathrm{F} 1-\mathrm{F} 4$ as predictors of the interneuron type would be an interesting problem to tackle with stacked classification or classifier chains. 
As a more flexible and less subjective way to characterize the interneurons, the categorical axonal features F1-F4 might be replaced with real-valued measures: we could, for example, measure the percentage of the axon that leaves the soma's layer instead of categorically distinguishing between 'intralaminar' and 'translaminar' axons. We are therefore developing an algorithm for such objective measurement of neuronal features F1-F4.

Finally, our data consisted of neurons from several species. While it is considered that the same morphological types of neurons exist in these species, there still might be inter-species variability. In the near future, we plan to further analyze the data to take this possibility into account. Also, although our data contained seven interneuron types, three of them - chandelier, neurogliaform, and horse-tailwere represented with fewer than five cells. Since there is medium to high inter-expert consensus on the definitions of those types, it would be interesting to repeat the present analysis with more cells of these types.

\section{Information Sharing Statement}

All used data-the 237 interneuron cell reconstructions and the corresponding experts' characterizations according to features F1 to F6-are available at http://cig.fi. upm.es/bojan/gardener/. The bnclass i fy R package will be made available on the CRAN repository (http://cran. r-project.org/) by the end of 2014 whereas the remaining software used is publicly available: caret and RWeka on CRAN and weka at http://www.cs.waikato.ac.nz/ml/weka/.

Acknowledgments This work was supported by grants from the following entities: the Spanish Ministry of Economy and Competitiveness (grants TIN2013-41592-P to B.M., C.B., and P.L.; BFU2012-34963 to J.DF.), CIBERNED CB06/05/0066 to J.DF., the Cajal Blue Brain Project (C080020-09; the Spanish partner of the Blue Brain Project initiative from EPFL) to B.M., C.B., J.DF., and P.L., and the European Union Seventh Framework Programme (FP7/2007-2013) under grant agreement no. 604102 (Human Brain Project) to C.B., J.DF., and P.L. R.B.-P. was supported by the Spanish Ministry of Economy and Competitiveness (CSIC).

\section{References}

Ascoli, G.A., Donohue, D.E., Halavi, M. (2007). Neuromorpho.org: a central resource for neuronal morphologies. The Journal of Neuroscience, 27(35), 9247-9251.

Ascoli, G.A., Alonso-Nanclares, L., Anderson, S., Barrionuevo, G., Benavides-Piccione, R., Burkhalter, A., Buzsaki, G., Cauli, B., DeFelipe, J., Fairén, A., et al. (2008). Petilla terminology: nomenclature of features of GABAergic interneurons of the cerebral cortex. Nature Reviews Neuroscience, 9(7), 557-568.

Bielza, C., \& Larrañaga, P. (2014). Discrete Bayesian network classifiers: a survey. ACM Computing Surveys, 47(1), 5:1-5:43.
Bielza, C., Li, G., Larranaga, P. (2011). Multi-dimensional classification with Bayesian networks. International Journal of Approximate Reasoning, 52(6), 705-727.

Breiman, L. (1996). Bagging predictors. Machine Learning, 24(2), $123-140$.

Cauli, B., Audinat, E., Lambolez, B., Angulo, M.C., Ropert, N., Tsuzuki, K., Hestrin, S., Rossier, J. (1997). Molecular and physiological diversity of cortical nonpyramidal cells. The Journal of Neuroscience, 17(10), 3894-3906.

DeFelipe, J., López-Cruz, P.L., Benavides-Piccione, R., Bielza, C., Larrañaga, P., Anderson, S., Burkhalter, A., Cauli, B., Fairén, A., Feldmeyer, D., et al. (2013). New insights into the classification and nomenclature of cortical GABAergic interneurons. Nature Reviews Neuroscience, 14, 202-216.

Dougherty, J., Kohavi, R., Sahami, M. (1995). Supervised and unsupervised discretization of continuous features. In Machine learning: proceedings of the twelfth international conference (pp. 194202).

Fairén, A., Regidor, J., Kruger, L. (1992). The cerebral cortex of the mouse (a first contribution-the 'acoustic' cortex). Somatosensory \& Motor Research, 9(1), 3-36.

Friedman, N., Geiger, D., Goldszmidt, M. (1997). Bayesian network classifiers. Machine Learning, 29, 131-163.

Glaser, J.R., \& Glaser, E.M. (1990). Neuron imaging with Neurolucida-a PC-based system for image combining microscopy. Computerized Medical Imaging and Graphics, 14(5), 307-317.

Glaser, E.M., \& McMullen, N.T. (1984). The fan-in projection method for analyzing dendrite and axon systems. Journal of Neuroscience Methods, 12(1), 37-42.

Gupta, A., Wang, Y., Markram, H. (2000). Organizing principles for a diversity of GABAergic interneurons and synapses in the neocortex. Science, 287(5451), 273-278.

Hall, M. (2007). A decision tree-based attribute weighting filter for naive Bayes. Knowledge-Based Systems, 20(2), 120-126.

Hall, M., Frank, E., Holmes, G., Pfahringer, B., Reutemann, P., Witten, I.H. (2009). The WEKA data mining software: an update. SIGKDD Explorations Newsletter, 11(1), 10-18.

Hand, D.J., \& Yu, K. (2001). Idiot's Bayes-not so stupid after all? International Statistical Review, 69(3), 385-398.

Hornik, K., Buchta, C., Zeileis, A. (2009). Open-source machine learning: R meets Weka. Computational Statistics, 24(2), 225232.

Kawaguchi, Y. (1993). Physiological, morphological, and histochemical characterization of three classes of interneurons in rat neostriatum. The Journal of Neuroscience, 13(11), 49084923.

Kuhn, M., Wing, J., Weston, S., Williams, A., Keefer, C., Engelhardt, A., Cooper, T. (2013). caret: classification and regression training. R package version 5.17-7.

Langley, P., \& Sage, S. (1994). Induction of selective Bayesian classifiers. In Proceedings of the 10th conference on uncertainty in artificial intelligence (pp. 399406): Morgan Kaufmann.

Maccaferri, G., \& Lacaille, J.C. (2003). Interneuron diversity series: hippocampal interneuron classifications-making things as simple as possible, not simpler. Trends in Neurosciences, 26(10), 564571.

McMullen, N.T., Glaser, E.M., Tagamets, M. (1984). Morphometry of spine-free nonpyramidal neurons in rabbit auditory cortex. Journal of Comparative Neurology, 222(3), 383-395.

Mihaljevic, B., Bielza, C., Larrañaga, P. (2013). bayess lass: an R package for learning Bayesian network classifiers. In Proceedings of useR! - the R user conference (p. 53). 
Mihaljević, B., Benavides-Piccione, R., Guerra, L., DeFelipe, J., Larrañaga, P., Bielza, C. (2014). Classifying GABAergic interneurons with semi-supervised projected model-based clustering. Artificial Intelligence in Medicine, (in press).

Minsky, M. (1961). Steps toward artificial intelligence. Transactions on Institute of Radio Engineers, 49, 8-30.

Morales, D., Vives-Gilabert, Y., Gómez-Ansón, B., Bengoetxea, E., Larrañaga, P., Bielza, C., Pagonabarraga, J., Kulisevsky, J., Corcuera-Solano, I., Delfino, M. (2013). Predicting dementia development in Parkinson's disease using Bayesian network classifiers. Psychiatry Research: NeuroImaging, 213, 92-98.

Panico, J., \& Sterling, P. (1995). Retinal neurons and vessels are not fractal but space-filling. Journal of Comparative Neurology, 361(3), 479-490.

Pearl, J. (1988). Probabilistic reasoning in intelligent systems. San Francisco: Morgan Kaufmann.

Peters, A., \& Jones, E.G. (1984). Cerebral cortex: volume 1: cellular components of the cerebral cortex. New York: Plenum Press.

R Core Team. (2012). R: a language and environment for statistical computing. Vienna: R Foundation for Statistical Computing.
Raykar, V.C., Yu, S., Zhao, L.H., Valadez, G.H., Florin, C., Bogoni, L., Moy, L. (2010). Learning from crowds. The Journal of Machine Learning Research, 11, 1297-1322.

Sadler, M., \& Berry, M. (1983). Morphometric study of the development of Purkinje cell dendritic trees in the mouse using vertex analysis. Journal of Microscopy, 131(3), 341-354.

Smialowski, P., Frishman, D., Kramer, S. (2010). Pitfalls of supervised feature selection. Bioinformatics, 26(3), 440-443.

Somogyi, P., Tamás, G., Lujan, R., Buhl, E.H. (1998). Salient features of synaptic organisation in the cerebral cortex. Brain Research Reviews, 26(2), 113-135.

Yang, Y., \& Webb, G.I. (2003). Weighted proportional k-interval discretization for naive-Bayes classifiers. In Advances in knowledge discovery and data mining (pp. 501-512). Springer.

Yang, Y., Webb, G.I., Wu, X. (2010). Discretization methods. In Data mining and knowledge discovery handbook (pp. 101-116). Springer.

Zaidi, N.A., Cerquides, J., Carman, M.J., Webb, G.I. (2013). Alleviating naive Bayes attribute independence assumption by attribute weighting. Journal of Machine Learning Research, 14, 19471988. 